\title{
Corela
}

Cognition, représentation, langage

HS-22 | 2017

Prédication et prépositions en anglais

\section{Verbes et constructions verbales en grammaire cognitive : la prédication autrement}

Jean-Rémi Lapaire

\section{OpenEdition}

Journals

Édition électronique

URL : http://journals.openedition.org/corela/4992

DOI : $10.4000 /$ corela.4992

ISSN : $1638-573 \mathrm{X}$

Éditeur

Cercle linguistique du Centre et de I'Ouest - CerLICO

Référence électronique

Jean-Rémi Lapaire, « Verbes et constructions verbales en grammaire cognitive : la prédication autrement », Corela [En ligne], HS-22 | 2017, mis en ligne le 31 août 2017, consulté le 01 mai 2019. URL : http://journals.openedition.org/corela/4992 ; DOI : 10.4000/corela.4992

Ce document a été généré automatiquement le 1 mai 2019.

\section{(c) (1) (3) (-)}

Corela - cognition, représentation, langage est mis à disposition selon les termes de la licence Creative Commons Attribution - Pas d'Utilisation Commerciale - Partage dans les Mêmes Conditions 4.0 International. 


\title{
Verbes et constructions verbales en grammaire cognitive : la prédication autrement
}

\author{
Jean-Rémi Lapaire
}

\section{Introduction}

1 La grammaire cognitive entretient une vision à la fois dramaturgique et constructionnelle du verbe. Dramaturgique, parce que le verbe permet de scénariser les événements et de signaler l'angle sous lequel ces derniers sont perçus. Constructionnelle, parce que faire usage d'un verbe dans le discours, c'est nécessairement l'intégrer à un cadre formel qui distribue des rôles et génère du sens. Concrètement, cela revient à identifier des actants, à valoriser ou à minorer certains protagonistes, à relier ces derniers entre eux, à adopter un point de vue et à inscrire l'ensemble dans un décor et une réalité (Langacker, 2000 : 24-26). Ecrire Their breaths fogged the window (texte à l'étude, 1. 30), c'est transformer la globalité d'un phénomène perceptuel (la buée répandue par l'haleine des passagers) en scène. C'est utiliser une structure verbale transitive pour définir des actants (participants) et organiser leur interaction: their breaths acquiert ainsi le statut d'agent, tandis que fog caractérise le mode d'agir et oriente l'énergie vers the window (second actant, affecté).

Ce qui est vrai d'une construction transitive simple $\left(\mathrm{SVO}_{\mathrm{D}}\right)$ est extensible à toute construction verbale, quelle qu'en soit la complexité. L'énonciateur choisit de mettre en syntaxe une expérience ${ }^{1}$. Pour cela, il puise dans un répertoire de formes conventionnelles, lexicales et grammaticales. Les signes grammaticaux qui sont impliqués dans ce processus de représentation et d'expression participent de plein droit à la fonction symbolique du langage. En outre, les constructions mobilisées dans le discours individuel préexistent à l'état de patrons structuraux (patterns) et de schèmes sémantiques. Autrement dit, les constructions ne sont pas générées ou composées dans l'instant de la parole, mais convoquées et utilisées comme autant de patrons d'agencement des unités et d'élaboration du sens. 
3 Pour prendre toute la mesure de ce que ces principes impliquent, nous commencerons par définir le statut des constructions verbales dans la grammaire cognitive générale de Langacker (2008). Nous montrerons ensuite que la grammaire des constructions, émergente chez Whorf (1942), amorcée par Lakoff (1987), formulée par Fillmore et Kay (1987), lancée par Goldberg (1995) et enfin « radicalisée » par Croft ${ }^{2}$ (2001), n'est qu'un développement syntaxique logique des « fondements » posés par Langacker $(1987,1991)$. La présentation est accompagnée d'exemples, tour à tour empruntées aux auteurs cités et au texte commun de référence.

\section{La grammaire cognitive de Langacker}

4 Le cadre théorique construit depuis 1987 par Ronald Langacker repose sur une idée à la fois simple et stable: la grammaire permet de réaliser des assemblages d'unités symboliques $^{3}$ (symbolic assemblies) de complexité variable, à partir de marqueurs ( markers) et de patrons structuraux (patterns) régis par un ensemble de conventions d'usage.

Most of the expressions we employ are symbolically complex, to some degree analyzable into smaller symbolic elements. Grammar consists of the patterns for constructing such expressions. (Langacker, $2008: 161$ ) [Notre soulignement]

5 Le processus d'assemblage s'applique autant au lexique qu'à la grammaire, ce qui interdit qu'on sépare les deux domaines :

$\mathrm{CG}^{4}$ makes the fundamental and highly controversial claim that grammar is symbolic in nature. More specifically, it holds that grammar and lexicon form a continuum residing exclusively in assemblies of symbolic structures. (Langacker, 2008 : 161) [En gras dans l'original]

Ainsi, la combinatoire qui est à l'œuvre dans une simple dérivation lexicale de type water > water-y et blind > blind-ness (1. 22) n'est pas fondamentalement différente de celle qui opère au niveau syntaxique dans l'énoncé qui l'accueille: There was a moment of watery blindness till the truck had dropped behind. Dans l'un et l'autre cas, il y a simultanément assemblage d'unités symboliques et assignation de sens.

7 L'ensemble des agencements possibles dans une langue (assemblies of symbolic structures) forme un répertoire identifiable et analysable de constructions que la grammaire reçoit pour mission d'inventorier et d'élucider.

Constructions are symbolic assemblies. The objective of grammatical analysis is to describe such assemblies in clear and precise detail. (Langacker, $2008: 161$ ) [Notre soulignement]

8 Reprenant l'énoncé cité plus haut, There was a moment of watery blindness (1. 22), nous décelons au moins trois constructions syntaxiques: existentielle (THERE BE NP), partitive-quantitative $(\mathrm{A}(\mathrm{N}) \mathrm{N}$ DURATION UNIT $\mathrm{OF} \mathrm{NP})$, modificative $(\mathrm{ADJ} .+\mathrm{N})$. Celles-ci fonctionnent bien comme des patrons d'assemblage d'unités et de configuration du sens. Pareille conception «configurationnelle» de la grammaire (Victorri, 2004: 77) prend nommément le contrepied du générativisme :

9 - les marqueurs syntaxiques (grammatical markers) font partie du symbolisme langagier ${ }^{5}$ de plein droit. Ils ont une valeur identifiable et ne sont jamais réductibles au statut d'outils dans une mécanique abstraite de génération syntaxique ${ }^{6}$. There dans There wasn't much traffic (l. 13), do dans I don't mind a little rain (l. 11), be et-ed dans You're focused on... (l. 27) devront ainsi être analysés comme des morphèmes grammaticaux « schématiques » ( 
schematic) mais « signifiants » (contentful, meaningful).

- les agencements grammaticaux (grammatical constructions) ne procèdent pas par transformation ou combinaison mais par intégration dans des patrons préexistants ( patterns), qui sont autant de structures symboliques organisatrices de représentations. On peut ainsi faire l'hypothèse qu'il existe un schème constructionnel modal qui informe l'ensemble de l'énoncé Maybe you should put on your glasses (1. 25). Ce schème, qu'une analyse plus complète permettrait de décomposer en sous-schèmes, associe à la structure syntaxique Maybe $\mathrm{X}_{\text {SUJET }}$ should $\mathrm{Y}_{\text {PREDICAT }}$ la structure sémantique < Speaker KINDLY ADVISES X TO PERFORM $Y>$ qui pragmatiquement s'interprète comme <Speaker ATTEMPTS TO MANIPULATE X INTO PERFORMING Y> (pour X sujet de rang 2). De même, l'interprétation de l'expression quantitative a gust of wind (blew up) (l. 16) repose sur l'existence d'un schème constructionnel associant l'agencement grammatical $\mathrm{A}(\mathrm{N}) \mathrm{X}$ of $\mathrm{Y}$ à l'interprétation $<\mathrm{A}(\mathrm{N})$ INSTANCE OR MANIFESTATION of Y> (également à l'œuvre dans des tours institués comme $a$ roar of laughter, a piece of evidence). Aucune structure profonde n'a ici besoin d'être postulée derrière l'énoncé de surface ${ }^{7}$ même lorsque celui-ci semble idiomatique. Les règles de la grammaire ne sont pas des règles de production mais d'appariement forme / sens: grammatical rules are patterns in the formation of symbolically complex expressions (Langacker, $2008: 23$ ) [Notre soulignement]. Cette idée est le fondement même de la grammaire cognitive des constructions examinée en 3.

- les catégories grammaticales (grammatical classes), qui incluent les parties du discours, ne sont pas purement formelles. Elles reposent sur des "archétypes conceptuels » ( conceptual archetypes), issus de la cognition et de l'expérience socio-physique générales. Les noms, par exemple, permettent de symboliser des unités d'expérience et de s'y référer comme à des choses: "For nouns, the archetype functioning as category prototype is the conception of a physical object » (Langacker $2008: 103)$.

Pour Langacker (2008: 108-112), le «schème » qui structure la catégorie du verbe (verb schema) repose sur une double capacité cognitive (cognitive ability) :

11 - la capacité à mettre en relation les actants (focal participants) impliqués dans un même événement (event);

- la capacité à inscrire l'événement dans le déroulé du temps (apprehending and tracking through time).

12 La seconde capacité retient plus particulièrement l'attention de Langacker (2008), qui oppose deux modes de saisie des événements : parcours sommatif (summary scanning) et parcours séquentiel (sequential scanning). Le premier type de saisie agrège l'ensemble des moments d'une scène verbale. Tout en étant mentalement récupérables dans leur déroulé, ces «états constitutifs " (component states) sont appréhendés en mode égalitaire et synthétique ${ }^{8}$. C'est notamment le cas des formes infinitivales et participiales. Les premières imposent une "vision globale et sommative" des états constitutifs de l'événement (a holistic and summative view), sans isoler de moment particulier: They were supposed to stay at the beach a week (l.1). Les secondes, participiales, permettent d'opérer une saisie atemporelle de l'événement, en adoptant une perspective interne (internal perspective) et non bornée pour -ing ou une post-perspective pour -ed/-en (posterior vantage point). Par exemple, dans Sarah sat next to him, leaning her head against the side window (1. 2), la participiale en -ing n'évoque en elle-même ni déroulement, ni durée, seulement une masse d'états constitutifs homogénéisés (a mass-like, internally homogeneous relationship). Pour Langacker, -ing indique que le point de vue sur l'événement (construal) est global-synthétique (holistic). Le contenu du prédicat est massifié et lissé, comme celui 
d'un nom indénombrable": Leaning her head against the side window probably meant something comparable à Her silence probably meant something [notre manipulation]. Quant à la post-perspective indiquée par-ed/-en, elle est patente dans parked beneath the underpass (1. 92), où la vision donnée du motocycliste arrêté est celle d'un homme ayant déjà garé son véhicule pour s'abriter de la pluie torrentielle.

Le second type de saisie, séquentielle, suit les étapes d'un état ou d'une action qui se déploie dans le temps (sequential viewing). Cette vision mentale de l'événement comme un processus se rapproche davantage de la perception visuelle des événements du monde ${ }^{10}$. Langacker (2008) propose ici une conception originale de la copule ou de l'auxiliaire be. Pour lui, ce verbe est foncièrement dynamique et séquentiel car il permet d'inscrire un état ou une série d'états dans le déroulé du temps et de les parcourir mentalement. Les effets interprétatifs les plus courants sont la propriété permanente (He was a tall, pale, grey-eyed man [1. 7]) ou transitoire (They were quiet a moment [1. 68]), le processus (I'm trying to be [a comfort] [1.62]), l'inscription dans la durée, etc. Si le narrateur avait écrit She was leaning her head against the side window, l'effet "scène du passé considérée dans son déroulement " aurait résulté du parcours séquentiel effectué par be sur l'ensemble des états représentés par V-ing. De même, l'interprétation «situation temporaire affectée de durée » de [The motorcyclist (1. 39)] was parked beneath the underpass serait l'œuvre de be.

Langacker (2008) estime qu'un verbe a pour vocation première de représenter un processus. Seule les formes (finies) ou les constructions qui permettent une saisie séquentielle de l'événement sont à proprement parler verbales. Les infinitifs, les participes (-ing/-ed), qui sont l'indice d'une saisie sommative, non processuelle de l'événement, ne sont donc pas des formes verbales, ou ont cessé de l'être, ${ }^{11}$ ce qui explique la facilité avec laquelle ces formes non finies du verbe peuvent acquérir un fonctionnement nominal (It's crazy to ride a motorcycle on a day like today; Putting on my glasses would help you to see?) ou adjectival (a blank, startling second ; a lulling sound ; their gleaming surfaces, a darkened look) $(1.40,26,33,15,59,18)$.

En résumé, chaque type de construction verbale reflète une perspective particulière adoptée sur l'événement. Langacker trace un parallèle intéressant entre l'expérience visuelle et la conceptualisation du réel (2000: 204-210). Le sujet cognitif (conceptualizer, cognizing subject) est comparé à un observateur (viewer) qui appréhende une "scène » ( scene) et ses «actants» (participants) dans une certaine "configuration " (viewing arrangement). Les formes choisies pour figurer l'événement sont fonction de la distance, de l'étendue (scope), du point de vue (vantage point), de la zone d'attention focale (focus, onstage region), de l'existence de figures (figures) se détachant d'un arrière-plan ( background), de la hiérarchisation entre éléments repères (landmarks) et éléments repérés (trajectors), du type de saisie (construal) privilégié. Il en découle que l'analyse d'une construction verbale nous renseigne autant sur l'événement que sur la façon dont cet événement est appréhendé par un sujet parlant et pensant. De façon plus générale, tout agencement de symboles langagiers donne simultanément accès aux objets de conception (objects of conception) du sujet et aux opérations de représentation dont procèdent lesdits objets.

\section{L'hypothèse de Whorf à l'épreuve de la prédication}

Les écrits de Benjamin Lee Whorf (1897-1941) ont exercé une influence déterminante dans l'émergence de la linguistique cognitive. Malgré la réévaluation critique de ce 
«penseur complexe » (a complex thinker) par George Lakoff dans Women, Fire and Dangerous Things (1987 : 328-369), Whorf a quasiment disparu des index et des bibliographies en grammaire cognitive. On trouve encore de timides allusions à Whorf chez Bernd Heine (1997) qui l'invoque pour défendre un compromis entre relativisme et universalisme. ${ }^{12}$ Cinq ans plus tard, John Taylor (2002) ne cite plus Whorf que de manière fugace dans sa volumineuse Cognitive Grammar, alors que l'ouvrage entend présenter la théorie au grand public universitaire, de façon synthétique et accessible. Mais l'éclipse est imminente: William Croft et Alan Cruse (2004), Günter Radden et René Dirven (2007) et enfin Ronald Langacker (2008) ne se donnent plus la peine d'invoquer Whorf. Même Evans (2012), dans son «survol» (overview) du cadre cognitiviste, ne cite pas Whorf directement, tout en reconnaissant que la linguistique cognitive dans son entier peut être qualifiée de «néowhorfienne » (neo-Whorfian) $)^{13}$.

17 Il y a là un paradoxe qui peut s'expliquer par le succès dévastateur de l'« hypothèse SapirWhorf » (the Sapir-Whorf hypothesis). Abusivement résumée et simplifiée, cette hypothèse a facilité la diffusion rapide et universelle des idées de Whorf tout en précipitant leur relégation en Occident. Selon cette hypothèse, rappelons-le, les notions et catégories que les langues lexicalisent ou grammaticalisent, non seulement découperaient ${ }^{14}$ et analyseraient le réel de façon variable, mais conditionneraient la perception et la cognition générale des locuteurs.

Every language is a vast pattern system, different from others in which are culturally ordained the forms and categories by which the personality not only communicates, but also analyzes nature, notices or neglects types of relationships and phenomena, channels his reasoning and builds the house of consciousness. (Whorf, $1956: 252$ )

Ce n'est pas l'idée de « relativisme linguistique » (linguistic relativism) qui a pu faire débat, puisque la variabilité des «découpages» (chopping up) et des procédures de "classification de l'expérience» (Boas, 1940:354) ${ }^{15}$ est une donnée objectivable. C'est plutôt le conditionnement de la perception et de la pensée par les formes particulières des langues qui s'est heurté à la biologie du corps, du cerveau et du langage. Puisque la physiologie de la perception est universelle, puisque la faculté de langage est non seulement « instinctive » (Pinker, 1994) mais ancrée dans un cerveau donné en partage à l'humanité (Jackendoff 1993), comment défendre la relativité linguistique de la perception? Poliment minorée ou agressivement écartée par les porteurs de conceptions innéistes du langage et universalistes et de la cognition, l'« hypothèse Sapir-Whorf » n'a pas encore été soumise à toutes les expérimentations et vérifications empiriques qu'elle mériterait, comme le suggère Lucy (1996) :

The possibility that the language we speak influences the way we think has excited both popular and scientific imagination in the West for well over a century [...] Yet this intense interest and manifest significance have not led to a commensurate volume and quality of empirical research. We still know little about the connections between particular language patterns and mental life - let alone how they operate. (Lucy, $1996: 37$ )

19 La pensée de Whorf n'a pas non plus alimenté la réflexion syntaxique des grammairiens cognitivistes autant qu'on aurait pu l'imaginer. Anticipant de façon visionnaire sur la grammaire des constructions (examinée dans la partie suivante), Whorf voit dans les agencements grammaticaux des matrices de configuration de la pensée et de génération du sens. Ce phénomène, qu'il nomme patternment, intègre les niveaux formel et cognitif. Plaçant les constructions syntaxiques et non les seuls mots au cœur du 
fonctionnement de la langue, Whorf énonce le principe fondamental suivant: The patterns of sentence structure that guide words are more important than the words (1956:253). Ce sont les patrons d'agencement formel et conceptuel ${ }^{16}$ (patterns) qui modèleraient le sens, non les unités :

Because of the systematic, configurative nature of higher mind, the "patternment" aspect of language always overrides and controls the "lexation" or name-giving aspect. Hence the meanings of specific words are less important than we fondly fancy. Sentences, not words, are the essence of speech, just as equations and functions, and not bare numbers, are the real meat of mathematics. (Whorf, 1956 : 258) [Notre soulignement]

Les structures empruntées par la langue pour combiner ses unités (combinatory schemes) sont à la fois normées (car calibrées par la grammaire) et signifiantes (car impliquées dans la construction du sens). Lorsque ces structures sont de type syntaxique (schemes of sentences, designs of sentence structure), elles ne doivent pas être conçues comme de simples lieux d'insertion et de liage car elles incorporent des éléments de représentation et font partie, à ce titre, du fonctionnement symbolique de la langue. La grammaire cognitive générale ne dit rien d'autre (cf. 1.) et la grammaire cognitive des constructions (cf. 3) se définit entièrement par rapport à ces "schèmes combinatoires" (Whorf, 1956: 253) qu'elle appelle constructions: ['Construction grammar' is] a framework that takes grammatical constructions as its primary units (Fillmore, 1988: 35); [G]rammatical constructions are the fundamental building blocks of language (Boas, $2014: 1$ ).

21 Whorf (1956: 265) prend un plaisir narquois à souligner que les langues européennes, anciennes ou modernes, ne sont pas exemptes de fantaisie ou d'incohérences logiques, alors qu'elles sont censées avoir porté la rationalité et la réflexion scientifique à leur plus haut degré. Inversement, plaide-t-il, certaines langues parlées par des sociétés réputées primitives ou préscientifiques (amérindiennes, zoulou, papoues) font preuve d'une étonnante précision descriptive et d'une admirable «intelligence grammaticale » ( grammatical finesse). Parmi les incohérences que Whorf épingle en grammaire anglaise figure l'homonymie constructionnelle frappant les verbes d'état, de perception et de mouvement. Le schéma de base de la transitivité (S-V-O) ferait ainsi entrer dans le moule de l'action transformative quantité d'événements qui ne sont pas des actions stricto sensu, comme « voir », « avoir », « tenir » ou « posséder. »

English pattern treats 'I hold it' exactly like 'I strike it', 'I tear it,' and myriads of other propositions that refer to actions effecting changes in matter. Yet 'hold' in plain fact is no action, but a state of relative positions. But we think of it, even see it, as an action, because language sets up the proposition in the same way as it sets up a much more common class of propositions dealing with movements and changes. We ASCRIBE action to what we call "hold" because the formula, substantive + verb $=$ actor + his action, is fundamental in our sentences. (262) [Notre soulignement]

Whorf va plus loin encore : la nécessité de distinguer un sujet engagé dans une action, conduit l'anglais (même scientifique) à traiter des phénomènes continus sur un mode analytique et à mettre en scène l'expérience sensorielle avec des actants. Par exemple, le phénomène perceptuel <éclat de lumière> devient <lumière engagée dans l'action de briller> [notre notation]. Dramatisée par la syntaxe, la lumière devient une entité isolable et agissante capable de faire et d'accomplir. La lumière brille, la lumière éclaire le mur, la lumière se répand dans la pièce. Nous n'y prêtons pas garde, mais nos constructions verbales scénarisent l'expression et donnent une syntaxe au réel qui n'est pas nécessairement la sienne : 
We are compelled in many cases to read into nature fictitious acting-entities simply because our sentence patterns require our verbs, when not imperative, to have substantives before them. We are obliged to say 'it flashed' or 'a light flashed,' setting up an actor IT, or a LIGHT, to perform what we call an action, FLASH. But the flashing and the light are the same; there is no thing which does something, and no doing. (Whorf, $1956: 263$ ) [Notre soulignement]

23 phénomène climatique <pluie> entrent dans le moule sémantico-syntaxique de l'action. Le vent se met à souffler, la pluie s'écrase et se répand. La syntaxe de l'anglais fait faire quelque chose aux éléments : au vent, au ciel chargé, à la pluie et à l'eau. Cela nous semble si évident, que nous oublions que cette mise en syntaxe d'événements perceptuels (perceptual events), avec ses actants placés dans des constructions (transitives ou intransitives) et enrôlés de force dans l'action, est plus symbolique que physique, plus culturelle que naturelle :

agir du ciel : the sky grew almost black (1. 9)

- agir du vent : Every now and then, a gust of wind blew up (1. 16)

- agir de l'eau: several enormous drops spattered the windshield...; The drops on the windshield grew closer together; Rain flattened the long, pale grass at the sides of the road; It slanted across the boat lots ; the rain stopped completely for a blank, startling second; the rain seemed to fall in sheets, layers and layers of rain beating down the cornstalks, flooding the rutted soil. (1. 9, 14, 16, 17, 32, 45)

\section{Vers une grammaire (cognitive) des constructions}

Bien que la parution de l'ouvrage intitulé A Construction Grammar Approach to Argument Structure (Goldberg 1995) soit considérée comme un moment décisif dans la propulsion et la diffusion internationales de la grammaire des constructions, l'opération a été préparée en amont par trois linguistes de Berkeley. Ces derniers ont nourri la jeune Adèle Goldberg de leurs intuitions et analyses tout au long de ses études doctorales : Paul Kay, Charles Fillmore et George Lakoff (qui fit soutenir en 1992 la thèse dont est issue la monographie de 1995).

L'étude détaillée des «constructions en there » par Lakoff (1987: 462-485) est l'occasion pour l'ex-sémanticien générativiste de proposer « une alternative aux théories établies de la grammaire ", qui associe "constructions grammaticales" et "modèles cognitifs " (462-463), en étant capable de rendre compte de tous les usages de langue, y compris idiomatiques. Au même moment, Fillmore (1988) présente à la Société Linguistique de Berkeley (BLS) une première tentative de formalisation des «mécanismes de la 'grammaire des constructions' «. Ce type de grammaire, écrit-il, traite les « constructions grammaticales » (grammatical constructions) comme des « unités premières » (primary units ) (35).

By grammatical constructions we mean any syntactic pattern which is assigned one or more conventional functions in a language, together with what is linguistically conventionalized about its contribution to the meaning or the use of structures containing it. (Fillmore, $1988: 36$ )

Cette définition, aussi délicate à comprendre qu'à manier, sera plus tard remplacée et standardisée par la formule lapidaire : « un appariement (conventionnel) entre forme et sens » (a [conventional] pairing of form and meaning). 
(...) all levels of grammatical analysis involve constructions : learned pairings of form with semantic or discourse function, including morphemes or words, idioms, partially filled and fully lexical patterns. This means that even the most general syntactic constructions have corresponding general rules of semantic interpretation (they are symbolic units). (Boas, $2014: 2$ )

Comme chez Whorf, les agencements formels sont intégrés de plein droit au processus de symbolisation, que la construction soit lexicale (lexical constructions) ou grammaticale ( grammatical constructions) :

In Construction Grammar, no strict division is assumed between the lexicon and syntax. Lexical constructions and syntactic constructions differ in internal complexity, and also in the extent to which phonological form is specified, but both lexical and syntactic constructions are essentially the same (...) : both pair form with meaning. (Goldberg, $1995: 7$ ) [Notre soulignement]

Les structures syntaxiques (syntactic frames) ne sont pas des assemblages neutres, des armatures formelles vides : elles signifient et font signifier en tant que formes langagières à part entière. Elles ont une valeur, elles génèrent, organisent et portent le sens ( contentful constructions), elles contribuent à définir le rôle des actants. Un exemple particulièrement éloquent est fourni par les «constructions résultatives » (resultative constructions), illustrées dans le texte de référence par : A mobile home's washed clear across that trailer park (1. 78). Goldberg (1995 : 188-198) montre comment l'insertion dans un schème constructionnel résultatif de verbes transitifs comme wipe, kick, paint, shoot (impliquant un changement d'état) ou intransitifs comme cough, cry, eat ou sleep, permet d'obtenir des énoncés clairement résultatifs : He wiped the table clean; She kicked the door open; He painted the house red; Harry shot Sam dead; She cried herself to sleep; He ate himself sick; She slept herself sober. Elle en identifie les contraintes et les restrictions, tout en proposant la modélisation suivante :

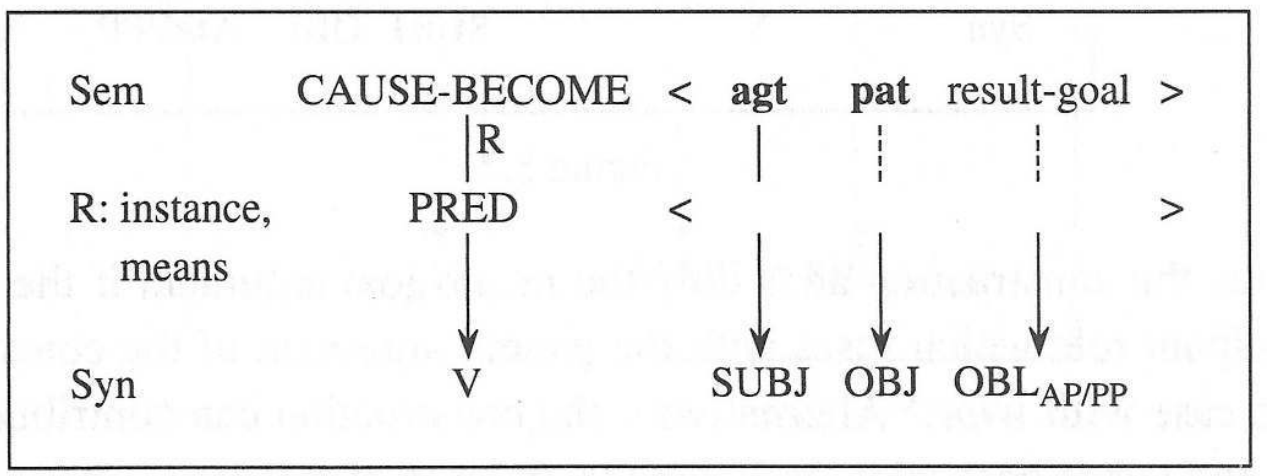

Figure 1 - La construction résultative (Goldberg, 1995 : 189)

Bien que la notion de construction soit extensible à l'ensemble de la langue, on note avec intérêt que la prédication occupe une place stratégique dans les premiers moments de la théorie. Ainsi, Lakoff (1987: 540-585) consacre plus d'une quarantaine de pages aux " constructions en there» (there-constructions), tandis que Fillmore (1988: 43-45) ébauche une représentation schématique généralisée de la "construction sujet-prédicat» en anglais (the subject predicate construction). Goldberg (1995) infléchit nettement l'axe de développement en direction de la structure argumentale (argument structure) que chaque verbe dessine et que les constructions achèvent de configurer, voire de reconfigurer. L'un des exemples fétiches de l'auteure, qui illustre l'influence du «sens constructionnel» (constructional meaning) sur l'interprétation d'un verbe, est celui de 
sneeze dans Sam sneezed the napkin off the table (29). Alors que le fonctionnement par défaut de sneeze est intransitif, alors que l'agentivité de son unique actant est incertaine, ce verbe peut devenir transitif, résolument agentif et causatif par insertion dans une construction à trois arguments appelée caused-motion construction. Celle-ci organise le sens de la manière suivante : 'X CAUSES $Y$ to MOVE $Z$ by sneezing' (9). Ce cas est loin d'être isolé, note Goldberg. Un énoncé comme My father frowned away the compliment and the insult (55) présente un phénomène similaire. Dans les deux cas, des éléments de sens, présents dans le verbe principal (sneeze, frown) sont "intégrés » à d'autres éléments de sens, véhiculés par la construction (et codés en petites majuscules dans les représentations). Goldberg (1995: 16) est ainsi conduite à énoncer le principe général suivant: "the meaning of an expression is the result of integrating the meanings of the lexical items into the meanings of constructions."

31 Le traitement que Goldberg propose du sens fait intervenir les notions de cadre (frame), de modèle cognitif idéalisé (idealized cognitive model) et de catégorisation radiale (radial categorization) synthétisés par Lakoff (1987). Elle en adapte seulement les principes à la sémantique du verbe (verb meanings): "Verbs, as well as nouns, involve rich frame-semantic meanings ; that is, their designation must include reference to a background frame rich with world and cultural knowledge" (Goldberg, 1995: 27). Pour parler en termes goffmaniens, l'expérience perceptuelle, sociale et culturelle que nous avons de sneeze ou frown est fondée sur un ensemble de scènes possédant chacune une "dramaturgie " spécifique (Goffman 1959: 240). Des rôles (participant roles) sont potentiellement définis qui contribuent à structurer la valence. Employer sneeze ou frown, ce n'est seulement nommer des actions : c'est construire des scènes qui s'inscrivent dans un cadre sociointeractionnel. Quels sont les implicites physiologiques et comportementaux associés à ces actions? Quels actants sont typiquement impliqués? Quels sont les éléments déclencheurs et les impacts de cette scène, autrement dit, quelle en est la structure causale?

Les éléments de «cadrage » sémantique, liés à l'expérience, ne sont pas seuls impliqués. Les propriétés sémantico-syntaxiques des constructions dans lesquels ces verbes s'insèrent exercent une importance tout aussi décisive sur leur interprétation. Goldberg (1995: 32-33) s'emploie donc à identifier le "sens constructionnel de base» (basic constructional meaning), « schématique » (skeletal) et « central » (central), des grands types de constructions verbales: transitive, intransitive et surtout bi-transitive. Ce sens est conçu comme flexible, extensible et modulable.

Constructions are typically associated with a family of closely related senses rather than a single, fixed abstract sense. [For example] ditransitive expressions in English typically imply that the agent argument acts to cause transfer of an object to a recipient. (Goldberg, 1995 : 31-32)

33 Ainsi, l'idée schématique dont est porteuse la construction bi-transitive- «transfert d'un objet vers un récipiendaire » (successful transfer of an object to a recipient) est-elle exploitée littéralement ou métaphoriquement par les verbes de création (Chris baked Jan a cake), d'engagement (Bill promised his son a car), d'autorisation ou de refus (Joe refused Bod a raise in salary). Comme Langacker (1987, 2000, 2008), Goldberg (1995) assigne aux agencements grammaticaux un «sens» (meaning) et une «valeur centrale » (central sense) compatible avec une variété de réalisations sémantiques. locuteurs natifs? Intuitivement, on peut envisager que ce soient les structures sémantico- 
syntaxiques associées à des scènes verbales courantes qui se schématisent et qui fournissent les patrons (patterns) dans lesquels d'autres verbes finissent par se glisser. Lorsque Sarah (texte à l'étude) suggère de garer la voiture au bord de la route et d'" attendre que l'averse passe » ("We could do that," Sarah said. "Stop and wait it out"), elle emploie une construction mêlant l'aspect (attendre jusqu'à la fin de l'épisode pluie), la causation (attendre et évacuer le danger) et enfin le but (attendre pour ne plus être menacé). Il y a dans ce wait it out, certainement un écho et peut-être une extension des "caused-motion constructions" (Goldberg, 1994: 160) plus primitives, directes et fréquentes, de type move, kick, throw it out. Encore faut-il être en mesure de le prouver. Goldberg, Casenhiser \& Sethuraman (2004) s'y emploient et montrent, études empiriques à l'appui, comment l'enfant parvient à acquérir les constructions ${ }^{17}$ de sa langue maternelle en reproduisant puis en opérant des " généralisations »: "children (...) go from specific knowledge of individual verb usage to knowledge of more general linking patterns, using general inductive strategies." (291). Se référant aux observations de Michael Tomasello (1992) sur le développement du langage enfantin, les auteurs soulignent l'importance des tournures verbales employées par les mères sur la création "d'îlots verbaux " (verb islands) et de « cadres » (frames) chez leurs enfants. Non contents de répéter, ces derniers insèrent ensuite, avec plus ou moins de bonheur, de nouvelles unités dans les structures qu'ils ont inconsciemment identifiées.

En interrogeant le corpus constitué par Bates, Bretherton et Snyder (1988), accessible via le système CHILDES (Child Language Data Exchange System) ${ }^{18}$, Goldberg et al. (2004: 295) notent le rôle pivot des constructions en go, get, give, throw, put, want, come dans la mise en place des agencements de base VL [V + Localisation] (she get in her bed, people come outside); VOL [V + Objet + Localisation ou trajectoire] (throw diaper away); VOo [V + Objet1 + Objet 2] (give me some milk). De façon plus intéressante encore, les chercheurs relèvent que chaque construction a son verbe installateur privilégié. Par exemple give pour la construction bitransitive VOO [X CAUSES Y TO RECEIVE Z], put pour la construction VOL [X CAUSES Y TO MOVE Z], go pour VL [X MOVES $\mathrm{P}_{\mathrm{PATH} / \mathrm{LC}}$ ] respectivement illustrées par Pat gave Chris a book, Marty put the milk in the fridge et I went to the store dans un discours d'adulte. À l'évidence, des verbes comme give, get, go sont des verbes de base de la langue, à emploi étendu (general purpose verbs), très présents dans le discours des adultes en interaction avec les enfants. Les scènes que ces "verbes premiers" (early verbs) nomment et organisent sont perceptibles et intelligibles dans le contexte immédiat d'énonciation. Autant de facteurs qui contribuent à en faire les vecteurs privilégiés de l'installation et de la généralisation des configurations formelles et sémantiques qu'ils véhiculent: The generality of the meanings of these verbs and their highly frequent and early appearance in children's speech suggests that they may aid children in generalizing patterns from the input (299).

Toute syntaxe et donc, toute grammaire est-elle constructionnelle ? La réponse la plus " extrême» est donnée par William Croft (2001) dans Radical Construction Grammar: constructions are the basic units of syntactic representation (4). 


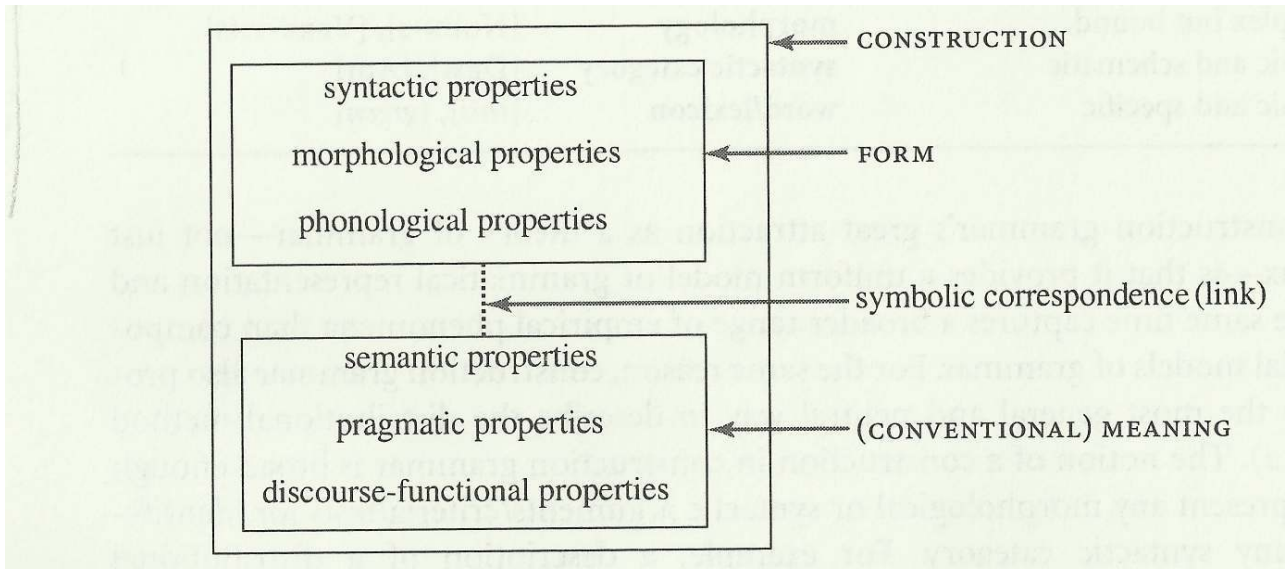

Figure 2 - Structure symbolique d'une construction (Croft, 2001 : 18)

Un peu plus loin, il précise : construction grammar has generalized the notion of a construction to apply to any grammatical structure, including both its form and meaning (17). Les tournures idiomatiques sont intégrées au modèle général de représentation des phénomènes ${ }^{19}$. Croft rejoint ici Langacker (1987) et sa conception de la grammaire comme inventaire de structures symboliques conventionnelles ${ }^{20}$. La boucle est bouclée : la grammaire cognitive des constructions est à la fois l'aboutissement syntaxique de la grammaire cognitive générale formalisée dès 1987 par Langacker et un spectaculaire retour aux fondamentaux de la théorie :

Grammar resides in patterns for the successive combination of symbolic structures to form more and more elaborate symbolic expressions. It is described by a structures inventory of "grammatical constructions." (Langacker, 1991 : 23)

\section{Conclusion}

La grammaire cognitive entretient une vision fondamentalement constructionnelle de la prédication. Refusant de réduire les formes verbales à des agencements purement formels, elle voit dans la morphologie flexionnelle ou l'auxiliarité autant d'indices des stratégies de conceptualisation du sujet parlant. Très attachée à caractériser les processus de conceptualisation, cette approche doit désormais affiner son traitement du sens en intégrant davantage les données socio-pragmatiques ${ }^{21}$.

Par son traitement intégré et synthétique du rapport forme / sens, la grammaire cognitive des constructions peut être mobilisée, de façon ludique et créative, dans l'enseignement des langues étrangères. Certaines structures gagnent en effet à être saisies en bloc, sans passer par une procédure longue et aléatoire de déconstructionrecomposition. Diverses expériences de "grammaire constructionnelle créative", auxquelles nous avons pu nous livrer à l'université, le suggèrent. Nous songeons à un travail récent sur la construction d'échange I don't mind doing a swap with you : your cow for these beans ${ }^{22}$ et la construction complexe Have you been such a fool, such an idiot as to give away my Milky-White, the best milker in the parish ${ }^{23}$ à la fois attributive, intensificatrice et causale. Au démontage fastidieux des agencements, en particulier ceux de la seconde construction, furent substituées des variations orales puis écrites sur les trames proposées. Le résultat dépassa toutes nos espérances : non seulement l'exercice stimula l'imagination et l'humour des jeunes anglicistes, mais l'approche créative se révéla 
propice à un engagement métalinguistique fort de leur part. Les sujets eurent en outre la satisfaction de produire des énoncés riches et authentiques. Loin de transformer la syntaxe en nomenclature, l'approche par constructions pourrait contribuer au renouvellement de la méthodologie d'enseignement et d'apprentissage de la grammaire à l'université. Nous espérons pouvoir le démontrer dans les années à venir.

\section{BIBLIOGRAPHIE}

Bates, E., Bretherton, I. \& Snyder L. (1988). From First Words to Grammar : Individual Differences and Dissociable Mechanisms. New York : Cambridge University Press.

Boas, F. (1940). Race, Language and Culture. New York : Macmillan.

Boas, H. (2014). "Cognitive construction grammar.” In Thomas Hoffmann (ed.) The Oxford Handbook of Construction Grammar. Oxford : OUP. Edition numérique consultée :

DOI : 10.1093/oxfordhb/9780195396683.013.0013

Croft, W. (2001). Radical Construction Grammar. Oxford : Oxford University Press.

Croft, W. \& Cruse D. A. (2004). Cognitive Linguistics. Cambridge : CUP.

Evans V. (2012). “Cognitive Linguistics.” Wiley Interdisciplinary Reviews (WIREs) : Cognitive Science, Volume 3, Issue 2, 2012. doi : 10.1002/wcs.1163

Fillmore, C. (1988). "The mechanisms of "Construction Grammar" BLS 14 : 35-55.

Fillmore, C. \& Kay, P. (1987). The goals of Construction Grammar. Berkeley Cognitive Science Report $\mathrm{N}^{\circ}$ 50. University of California at Berkeley.

Goldberg, A. (1995). A Construction Grammar Approach to Argument Structure. Chicago : The University of Chicago Press.

Goldberg, A., Casenhiser D. \& Sethuraman N. (2004). "Learning argument structure generalizations." Cognitive Linguistics 15-3 (2004), 289-316.

Heine, B. (1997). Cognitive Foundations of Grammar. New York : Oxford University Press.

Jackendoff. R. (1993). Patterns in the Mind. Language and Human Nature. New York : Harvester Wheatsheaf.

Lakoff, G. (1987). Women, Fire and Dangerous Things : What Categories Reveal about the Mind. Chicago : University of Chicago Press.

Langacker, R. (1987). Foundations of Cognitive Grammar. Volume 1. Theoretical Prerequisites. Stanford : Stanford University Press.

Langacker, R. (1991). Concept, Image and Symbol. The Cognitive Basis of Grammar. Berlin : Mouton de Gruyter.

Langacker, R. (2000). "Viewing in cognition and grammar." Grammar and Conceptualization. Berlin : Mouton de Gruyter, 203-246.

Langacker, R. (2008). Cognitive Grammar. A Basic Introduction. Oxford : Oxford University Press. 
Lucy, J. A. (1996). “The scope of linguistic relativity : An analysis and review of empirical research.” In J.J. Gumperz and S.C. Levinson (eds.), Rethinking Linguistic Relativity. Cambridge : Cambridge University Press, 37-69.

Pinker, S. (1994). The Language Instinct. London / New York : Penguin Books.

Radden, G. \& Dirven R. (2007). Cognitive English Grammar. Amsterdam : John Benjamins.

Taylor, J. (2002). Cognitive Grammar. Oxford : Oxford University Press.

Tomasello, M. (1992). First verbs : A Case Study of Early Grammatical Development. Cambridge : Cambridge University Press.

Tyler, A. (1985). The Accidental Tourist. London : Vintage Books, 1-5.

Victorri, B. (2004). « Les grammaires cognitives » In Catherine Fuchs (ed.), La linguistique cognitive, Paris / Gap : Ophrys, 73-98.

Whorf, B. (1956). "Language, Mind and Reality". In Language, Thought and Reality. Selected writings of Benjamin Lee Whorf. Cambridge, Mass : The MIT Press, 246-270.

\section{NOTES}

1. Notre formulation.

2. "Radical construction grammar is the syntactic theory to end all syntactic theories" (Croft, $2001: 4)$.

3. Langacker (2008: 5) définit "symbole" et "symbolique" de la façon suivante: «l'appariement entre une structure sémantique et une structure phonologique " (the pairing between a semantic structure and a phonological structure).

4. $C G$ : Cognitive Grammar

5. Bernard Victorri (2004: 77) souligne l'originalité et l'importance de cette position, notamment dans le contexte américain: "Le point clé, qui fait tout l'intérêt de cette approche, c'est de considérer que les aspects grammaticaux sont aussi porteurs de sens en eux-mêmes. " Langacker (1991 : 211) écrit lui-même : all grammatical units are claimed to be symbolic, and thus to have conceptual import.

6. L'un des combats intellectuels les plus ardents que Langacker ait pu mener est la reconnaissance du caractère signifiant (meaningful) de tous les morphèmes grammaticaux, sans exception. Même si leur «sens" (meaning) peut être très "schématique » (schematic) et donc délicat à caractériser, les marqueurs ne se contentent pas de coder des opérations syntaxiques, ils signifient : even the most 'grammatical' of grammatical markers-forms like be, do, of, the infinitival to, agreement markers, case inflections and derivational affixes-are viewed in CG as being meaningful (2008: 23).

7. Cognitive grammar does not posit deep or underlying syntactic structures which are distinct from surface structures. By and large, the morphemes overtly present in an expression coincide with the semantic units which determine its meaning (Langacker, $1991: 113$ ).

8. All the component states are simultaneously active and available. They form a single gestalt comparable to a multi-exposure photograph (Langacker, $2008: 111$ ).

9. La nominalisation complète en -ing ne fait que mener ce processus à terme: The hammering on the roof resumed; The only sound was the drumming of rain on the overhang far above them $(1.33,1.94)$.

10. Sequential scanning represents the actual nature of the real-time viewing experience, where just one component state is accessible at any given instant (Langacker, $2008: 111$ ).

11. The same content can be construed as either a process or a non-processual relationship, depending on whether it is accessed via sequential scanning or summary scanning. A basic proposal of CG is that a verb profiles a process. Sequential scanning is thus implied by categorization as a verb. When the same content 
is viewed in summary fashion, the resulting expression belongs to another grammatical category (e.g. an infinitive or a participle) (Langacker, 2008 : 112) [Notre soulignement].

12. Heine (1997 : 11) écrit ainsi : Evidence in favor of the universalist perspective is massive and has come from all major camps of modern linguistics. The relativist position is much harder to defend. The work of its main proponent, Benjamin Lee Whorf (1956), is not uncontroversial, to put it mildly; even fervent adherents of this position take care not to be associated too closely with Whorf's main thesis. Nevertheless, some intriguing evidence in favor of the relativist position has more recently become available. This evidence suggests that there are salient alternatives of conceptualization which human beings have developed and which influence the way languages are used and language is structured.

13. Evans (2012) note en effet: Cognitive linguists hold that [the] language-specific 'packaging' [of thoughts for communicative purposes] has profound consequences on nonlinguistic cognition. That is, language influences how we categorize aspects of our sociophysical environment, and how we think about reality, independently of language [...] This view is [...] a neo-Whorfian perspective on the relationship between language and nonlinguistic cognition.

14. Each language performs the artificial chopping up of the continuous spread of existence in a different way (Whorf, $1956: 253$ ).

15. L'anthropologue Franz Boas (1858-1942), qui fut l'un des maîtres à penser de Benjamin Lee Whorf, aux côtés d'Edward Sapir (1884-1939), voit dans la catégorisation environnementale et culturelle de l'expérience le principe fondateur des langues humaines: The classification of experience, which is the foundation of all linguistic expression does not follow the same principles in all Amerindian languages. On the contrary, many forms are found. The content of nouns and verbs depends upon cultural conditions (...) Terms of relationship and those relating to social structure vary in their contents; classifications occur such as animate and inanimate; long, flat or round; female and non female. In verbs, modalities of action, forms of object acting and acted upon, or local ideas may be expressed (Boas, 1940 : 354). [Notre soulignement]

16. Traduction générale que nous proposons de pattern chez Whorf.

17. Les auteurs redéfinissent les constructions en termes langackeriens de la manière suivante : correlations between formal linguistic patterns and meanings. (290)

18. De ce corpus sont extraites et annotées les productions de 13 garçons et 14 filles à 20 et 28 mois, ainsi que les productions de 15 mères à leur enfant de 28 mois.

19. Any construction with unique, idiosyncratic morphological, syntactic, lexical, semantic, pragmatic or discourse-functional properties must be represented as an independent node in the constructional network in order to capture the speaker's knowledge of their language (Croft, $2001: 25$ ).

20. Grammatical structure resides in symbolic units alone (Langacker, $1991: 20$ ).

21. Il pourrait par ailleurs être intéressant de mettre en regard le caractère conventionnel des schèmes constructionnels (constructional schemas / patterns) avec le caractère ritualisé de l'interaction communicative (conversational patterns).

22. Source : conte populaire, Jack and the Beanstalk. Syntaxe : Det possessif + N - FOR - SN. Sens de base < If $\mathrm{X}$ is given THEN $\mathrm{Y}$ should be given in return>. Sens pragmatique <IF someone gives you $X$, expecting $Y$ in return, THEN you should accept>. Productions étudiantes : YOUR bread FOR MY water. YOUR mobile phone FOR MY brother. YOUR body FOR MY soul. YOUR money FOR my children. YOUR religious beliefs FOR MY burger. YOUR kingdom FOR MY horse. MY kingdom FOR YOUR latte. MY toothpick FOR YOUR car. MY pants FOR YOURS.

23. Source : conte populaire, Jack and the Beanstalk. Productions étudiantes : What! Have you been such a jerk, SUCH AN incredible piece of shit, AS TO give me only vegetable soup for dinner...; What! Have you been SUCH A bitch, SUCH A slut, SUCH A ..., AS TO sleep with my boyfriend!; What ! Have you been SUCH A pervert, AS to spy on my sister in the shower ! 


\section{RÉSUMÉS}

Le terme "prédicat » est communément utilisé en grammaire cognitive, mais la prédication occupe une place marginale dans la théorie, qui préfère raisonner en termes de construction. La langue dispose en effet d'un répertoire d'agencements formels qui sont autant de structures, procédant par assemblage de symboles et appariements conventionnels forme-sens. Organisatrices et médiatrices de l'expérience qu'elles sont chargées de mettre en syntaxe, les constructions verbales scénarisent les événements, en distinguant cadres, repères, actants, et en mettant en perspective leur interaction. L'analyse d'une construction verbale renseigne donc autant sur l'événement lui-même que sur la façon dont ce dernier est appréhendé par un sujet parlant, percevant et pensant. En grammaire cognitive, toute forme ou catégorie, lexicale ou grammaticale, contribue à l'élaboration du sens. Aucune n'est arbitraire ni vide. Même la plus schématique s'avère signifiante.

Bien que les noms de Kay, Fillmore, Lakoff, Goldberg et Croft soient ceux volontiers associés à l'émergence et à la diffusion de la grammaire des constructions, il faut reconnaître l'influence capitale (mais rarement assumée) de Whorf, qui très tôt conçoit la syntaxe comme un patron structural et cognitif, une forme symbolique à part entière. Mais surtout, il est important de comprendre que la grammaire cognitive des constructions repose tout entière sur les principes fondamentaux de la grammaire cognitive générale que Langacker énonce dès les fondements.

Although the term predicate is routinely used in cognitive grammar, predication only plays a marginal role in the theory. Cognitive grammarians are more focused on the constructional frames that verbs fit into. Constructions are assemblies of symbolic structures, conventional pairings of meaning and form, which organize and mediate experience. Verb constructions allow speakers to frame events as scenes. A ground is set up, landmarks are established, participants identified, roles distributed, and interactions negotiated. Constructions reveal both content and construal-how the speaker-cognizer views and configures a scene. Cognitive Grammar treats all linguistic forms and categories-lexical and grammatical-as meaningful : none is empty, and all have conceptual import- even the most schematic.

The names of Kay, Fillmore, Lakoff, Goldberg and Croft are characteristically associated with the creation and international diffusion of construction grammar. Yet, Whorf's theory of patternment, which clearly unites form and construal, had a profound influence on the early developments of the theory. More importantly, it is essential to acknowledge that construction grammar rests entirely on the general founding principles of cognitive grammar, as spelled out by Langacker from the very start.

\section{INDEX}

Mots-clés : grammaire cognitive des constructions, verbes, structure argumentale, patrons structuraux, opérations de représentation

Keywords : cognitive grammar of constructions, verbs, argument structure, patterns, construal operations 


\section{AUTEUR}

\section{JEAN-RÉMI LAPAIRE}

EA CLIMAS, Université Bordeaux Montaigne

jrlapaire@u-bordeaux-montaigne.fr 